\title{
Exclude Laboratory Test Data From Statistics Indicator
}

National Cancer Institute

\section{Source}

National Cancer Institute. Exclude Laboratory Test Data From Statistics Indicator. NCI

Thesaurus. Code C119847.

Specifies whether the laboratory test result values should be excluded from the statistical analysis. 